\title{
Cambio organizacional: Una aproximación por valores
}

\author{
Díaz Barrios, Jazmín*
}

\section{Resumen}

La era actual se caracteriza por una búsqueda de la identidad del hombre, donde el pivote del cambio organizacional es el individuo; cambiar en este entorno depende, en gran medida, de los valores que imperen en la cultura de la organización. Este trabajo documental intenta identificar aquellos valores, condición sine-qua-non, para el logro de los objetivos de cambio y analizarlos a fin de determinar sus indicadores, con el objeto de presentar una herramienta a las organizaciones que les facilite adaptarse a los nuevos tiempos. Se encontró que los valores: Delegación, Comunicación, Colaboración, Participación y Aprendizaje, son esenciales en los cambios integrales de esta era. Se determinaron 20 indicadores de su presencia. Se concluye que con esta información cada ente puede elaborar instrumentos que le permitan saber si los valores requeridos están presentes y en caso contrario tomar decisiones que alineen a la organización alrededor de los valores humanistas planteados, incrementando así las posibilidades de éxito en el proceso de cambio y por ende, las posibilidades de supervivencia en esta nueva época.

Palabras clave: Cambio, cultura, organización, valores.

\section{Organizational Change: A Values Approach}

\section{Abstract}

These times are characterized by a search for the identity of man, where the axis of organizational change is the individual; and change in this environment largely depends on the values that rule the organizational culture. This bibliographical research attempts to identify those values which condition sine qua non the achievement of the objectives of change, and to analyze them in order to determine their indicators, in order to present a tool by which organizations can more easily adapt to a new era. It was found that the values: delegation, communication, collaboration, participation and learning, are essential to integral change in this era. The research determined the presence of 20 indicators. It was concluded that with this information, each organization can elaborate instruments designed to know if the required values are

\section{Recibido: 05-02-17. Aceptado: 05-10-16}

Dra. en Ciencias Gerenciales, Investigadora del Centro de Estudios de la Empresa de la Facultad de Ciencias Económicas y Sociales (FCES) de la Universidad del Zulia (LUZ) acreditada en el Programa de Promoción al Investigador (PPI) del Ministerio de Ciencia y Tecnología, Profesora de la Escuela de Administración y Contaduría Pública de FCES, LUZ.

E-mail: jdbconsult@cantv.net 
present, and if they are not, determine the manner in which to make take decisions that will align them around the proposed human values, incrementing in this way the possibility for success in the process of change, and thus the possibility for their survival in this new era.

Key words: Changes, culture, organization, values.

\section{Introducción}

En la literatura administrativa, es un tema bastante manido la necesidad de cambio en las organizaciones que pretenden ser competitivas e insertarse en el proceso de globalización. A la par, ha habido en las últimas décadas cambios sociales, económicos y culturales tan profundos que es lícito decir que vivimos una nueva fase histórica postmoderna. Autores como Buela (1997) plantean como característica fundamental de esta época la búsqueda y defensa insobornable de la identidad de los hombres y de los pueblos. Esto nos lleva a pensar, que aun con la presión de la competitividad, no solo importa el qué, sino también el cómo se lleva a cabo esos cambios.

Este cambio organizacional está representado por un conjunto de alteraciones tanto estructurales como de comportamiento en una organización (Chiavenato, 1995), pero no ocurre de manera espontánea, comienza con la presión de fuerzas exógenas (competencia, gobierno), fuerzas endógenas (desempeño laboral) o por una combinación de ambas. La modificación en las características de la fuerza laboral (nivel educativo, aspiraciones, necesidades familiares, valores) ha generado en los últimos tiempos una fuente endógena de cambio bastante fuerte. En esta época postmoderna, los individuos que conforman las organizaciones piensan, sienten y actúan diferente a sus predecesores.
Los modelos clásicos de cambio (estructural y tecnológico) no son ahora suficientes para lograr los cambios deseados. Comenta Martínez (2003) que el mundo de hoy se caracteriza por estar interconectado de manera tal que todos los fenómenos son recíprocamente interdependientes y que para describirlo necesitamos una perspectiva más amplia, holista y ecológica que no pueden ofrecernos las concepciones reduccionistas. Los modelos clásicos caen en esta categoría, de allí posiblemente su historial de fracasos en los últimos tiempos.

Gibson et al. (1996), plantean otra perspectiva que se sitúa, según ellos, a medio camino entre los dos enfoques antes mencionados: Reeducación. Explican los autores que la reeducación implica un conjunto específico de actividades en las que se reconoce, que ni el poder ni la razón por sí solos pueden generar el cambio anhelado. Ese conjunto de actividades es la esencia de lo que en las últimas tres décadas del pasado siglo se llamó Desarrollo Organizacional (DO) como modelo de cambio organizacional basado en las personas y que ahora a la luz de las ideas postmodernas, es simplemente la forma natural de cambiar organizaciones: Cambio en los patrones de conducta de los empleados, concentrándose en sus habilidades, actitudes, percepciones y expectativas en el ámbito individual, grupal y organizacional, a fin de trasladar a la organización entera a un nivel superior de funcio- 
namiento; es un cambio de la organización, a través de las personas, a través de sus valores y donde la estructura y la tecnología giran alrededor del hombre.

Se requiere conocer, en primer lugar, cuales son los valores que promueven la participación de los individuos en los cambios; y en segundo lugar, si esos valores están presentes en la organización estudiada. Lo anterior implica, por una parte, estudiar las características de las organizaciones en la postmodernidad para saber cuales valores están positivamente relacionados con ella; y por la otra, al conocer los valores, estudiarlos detalladamente para definir cuales son los indicadores de su presencia en una organización en particular. Este fue el trabajo que se llevó a cabo y se pretende con él, proporcionar a todas las organizaciones en esta nueva era, una guía que les permita diagnosticar.

\section{Cambio organizacional basado en las personas}

Desde los albores del DO, este modelo de cambio tuvo una definida base de valores humanistas, premisas específicas y alta sensibilidad ética (Bennis, 1973; Friedlander, 1976; Kahn, 1976). El DO se ocupó del bienestar de los participantes en el cambio organizacional y de facilitar su éxito. Ahora bien, si fue importante en el nacimiento de la Postmodernidad, en este siglo, más que importante, es imprescindible. Si bien es cierto que en los primeros tiempos Kahn (1976) criticó el DO por con- siderarlo un estudio de casos, y posiblemente tenía algo de razón, en la actualidad su análisis y utilización es general.

Estudios recientes (Wooten y White, 1999) en organizaciones envueltas en cambio organizacionales (reestructuraciones, reducción de tamaño organizacional, etc.) indican que la gente esta más dispuesta a aceptar el cambio visto como justo y a soportar la adversidad, cuando ellos ven los resultados equitativos provenientes de difíciles elecciones organizacionales. Dicho en otras palabras, cuando el personal percibe que la toma de decisiones esta basada en valores humanistas como la justicia y la equidad.

Diferentes autores han escrito sobre los valores organizacionales que deben imperar en los cambios exitosos. Margulies y Raia (1978) identificaron seis valores principales relacionados básicamente con el ambiente que se crea en la empresa para el personal: El trato como seres humanos complejos con un conjunto de necesidades a satisfacer, la posibilidad de desarrollar potencialidades, de influir con sus ideas en la organización, el trabajo excitante que represente retos y un incremento en la efectividad de la organización, fueron los elementos que encontraron en común en las experiencias exitosas.

Warwick y Thompson (1980), con una visión más externa, plantearon que encontrar las necesidades del cliente, guiarlo honestamente ${ }^{1}$, usar presunciones realistas sobre la gente y la organización y a partir de allí crear un ambiente de corrección, 
participación, apertura y confianza, eran la clave para que los cambios organizacionales lograran el éxito esperado. Por su parte, Gellerman et al. (1990) encontraron que los valores principales eran aquéllos que centraban la vida organizacional alrededor de justicia y equidad para todos sus participantes: a) Potencial humano y empowerment; b) Respeto, dignidad y darle valor a los individuos; c) Autenticidad, congruencia, honestidad y apertura en la aceptación de otros; d) Flexibilidad, cambio y proactividad; e) Aprendizaje, desarrollo, crecimiento y transformación; f) Actitudes ganar-ganar, cooperación, colaboración, confianza, comunidad y diversidad; g) Participación y democracia en la toma de decisiones y h) Efectividad, eficacia y alineación.

En otro estudio, Hurley et al (1992) efectuaron una encuesta a consultores empresariales, sobre los valores que consideraban principales y encontraron que para los agentes de cambio había cinco valores claves referidos a: delegación de autoridad para actuar; apertura en la comunicación, empleados que forman parte de los procesos, de los resultados y perciben tanto la colaboración como el aprendizaje continuo como positivos y valiosos. Estos resultados se convalidan con los de Gellerman et al. (1990) y Margulies y Raia (1978) en el sentido que los individuos disminuyen la resistencia al cambio y reaccionan positivamente, incluso apoyan los procesos de cambio, cuando se sienten parte del proceso y no simplemente peones en un tablero de ajedrez.

A su vez French y Bell (1996) apoyan estos postulados con su concepción de valores humanistas, optimistas y democráticos, basados en el respeto a la importancia y dignidad del individuo, su valía, potencial de crecimiento, desarrollo y bondad. Además de proclamar la justicia y equidad de poder.

Podría continuarse citando autores que expresan y defienden los valores humanistas como parte del proceso de cambio planeado en las personas (Brown y Dodd (1998), Burke (1997), Feurer y Chaharbaghi (1995), Harung y Dahl (1995), Lloyd y Kidder (1997) y London (1999); sin embargo esta revisión de antecedentes lo que pretende es dejar establecida la prelación entre valores humanistas y el modelo sociotécnico ${ }^{2}$. Es decir, cambiar integralmente la organización, requiere indefectiblemente la consideración de valores humanistas. Ahora bien, dando por sentado lo anterior, podrían surgir dudas sobre dos aspectos: En primer lugar, si es realmente necesario usar este modelo de cambio en particular en el entorno actual, frente a otros (estructural, tecnológico), que han sido tan utilizados en la modernidad. $Y$ en segundo lugar, de requerirse el modelo de cambio en las personas, cuáles serían los valores humanistas asociados, es decir, esos valores condición sine-qua-non, para el éxito del proceso de cambio planteado. (estructura, tecnología, procesos, operación) con el sistema humano (actitudes, percepciones, motivaciones, expectativas). 


\section{Cambios en el nuevo paradigma organizacional}

Estamos asistiendo a una transformación fundamental de nuestro modo de pensar, percibir y valorar, es decir, al nacimiento de un nuevo paradigma de la ciencia en cuanto a modo de conocer (Martínez, 2003).

En este sentido, Daft (1998) ha contrastado el nuevo paradigma organizacional postmoderno emergente con el paradigma organizacional moderno. El observó que las cualidades tradicionalmente consideradas igualitarias (igualdad, delegación, relaciones horizontales, construcción por consenso) son particularmente importantes en la organización postmoderna. Cada día emergen nuevos tipos de organizaciones, en respuesta a las condiciones caóticas y turbulentas del entorno. En este ambiente, los valores principales, premisas y principios éticos, eventualmente se actualizarán, más por la necesidad de sobrevivir que por la virtud misma.

Incluso el marco de referencia para el postmoderno DO teórico y práctico podría ser un marco de justicia social. Individuos participando en los cambios por convicción en vez de coerción debido a metas, políticas y procedimientos claros y sin ambigüedades, debido a un real interés por sus necesidades individuales, a una comunicación clara y honesta, a un desarrollo de sus potencialidades que le permita participar en las decisiones. Esto no es de extrañar, ya que gran parte de la tecnología actual en DO ha surgido en oposición a los modelos autocráticos, rígidos y excesivamente reglamentados en los cuales la justicia y la equidad estaban ausentes.

Agrega Daft (1998) que la organización postmoderna tendrá una estructura flexible y descentralizada con bordes difusos, comunicación informal, liderazgo servil, control a través de la autoregulación y con el igualitarismo como guía principal. En este ambiente, lo único que puede salvar a una organización es un cambio hacia una fuerte cultura, fuertes valores que la guíen por encima de sus líderes hacia las necesidades del cliente, que responda a sus obligaciones a través del autocontrol y la responsabilidad propia, que potencie a los trabajadores a desarrollar habilidades competitivas a través de su aprendizaje continuo. Los modelos estructurales no proveen cambios conductuales, solo un modelo de cambio centrado en las personas, se ocupa de desarrollar esas habilidades.

De igual manera, Grieves (2000) se suma a lo expuesto por Daft (1998), al manifestar que con el cambio de siglo, el modernismo le dio paso al post-modernismo. En el nuevo milenio la microtecnología ha incrementado la velocidad de la vida laboral, el redimensionamiento (downsizing), ha incrementado las redundancias y percepción de inseguridad en el tradicional sitio de trabajo. El trabajo flexible se ha incrementado, empleados a medio tiempo, independientes, con contratos temporales, han crecido. Todas estas características van, en aumento y están ligadas al aumento de la competencia en la economía global. Incertidumbre, ambigüedad y complejidad son características de las organizaciones post-modernas influenciadas por "alta tecnolo- 
gía", organizaciones virtuales y procesos intensivos de conocimiento.

En este entorno, el aprendizaje está inextricablemente unido al desarrollo organizacional, a fin de encontrar soluciones humanas a los problemas y beneficios resultantes de las siguientes situaciones: a) Cambio de estructuras organizacionales y la necesidad de lidiar con patrones e interrelaciones en constante cambio; b) Crecimiento de la diversidad cultural organizacional como resultado de los negocios globales y las alianzas estratégicas; c) Compañías de conocimiento que más que reaccionar, influencian el cambio con su pensamiento estratégico y d) Desarrollo de los recursos humanos como una iniciativa estratégica para el pensamiento creativo y la innovación.

Grieves (2000), agrega que las organizaciones actuales requieren una colaboración simbiótica para obtener beneficio mutuo; que el conocimiento laboral requiere experticia multidisciplinaria y aprendizaje colaborativo para compartir el dominio de ese conocimiento y redes estratégicas de comunicación e información para maximizar las oportunidades. La fluidez y transformación dependen del aprendizaje organizacional y no del entrenamiento; se requiere orientación a la excelencia a fin de convertirse en empresas clase mundial. En todo este contexto, la cultura organizacional tiene implicaciones en el liderazgo compartido y en las percepciones y motivaciones de los empleados.

Ahora bien, ¿cómo se logra que los individuos, grupos y las organizaciones como un todo, ahora interrelacionadas, tengan compromiso, sean colaboradoras, se orienten a la excelencia, practiquen el aprendizaje continuo y desarrollen redes de comunicación abiertas, para poder continuar siendo competitivos en el nuevo milenio? La respuesta esta en un modelo que provea aprendizaje organizacional $^{3}$, el cual sólo puede ser realizado por los miembros de esa organización al cambiar ellos hacia una cultura de aprendizaje, colaboración, compromiso, visión e información compartida: Un modelo humanista basado en esos valores.

En relación al segundo aspecto planteado, sobre cuáles serían esos valores indispensables para el éxito del modelo: Los dos autores anteriormente citados Daft (1998) y Grieves (2000), coinciden en la necesidad de una cultura de aprendizaje, colaboración, compromiso, comunicación y aprendizaje continuo, además de autocontrol y responsabilidad personal, entre otros.

Adicionalmente, Porras y Hoffer (1986), realizaron una investigación a fin de conocer los principales comportamientos efectivos, potencialmente asociados con resultados organizacionales positivos. Ellos preguntaron a 42 estudiantes y practicantes de DO de primera línea. Las respuestas de estas encuescas, valores, creencias y actitudes que incrementan la conservación, el crecimiento y el progreso de la organización" (Guns y Anundsen, 1996:16). 
tas, se enfocaron en dos grupos de comportamientos: uno para los miembros de la organización y otros para los gerentes solamente.

El instrumento orientado a toda la organización identificó nueve categorías de comportamiento asociadas con un desempeño excepcional. Las empresas cuyos miembros exhibían entre ellos con una alta frecuencia los comportamientos abajo mencionados, tenían mejor desempeño que los que no los poseían. Estas categorías fueron: apertura de comunicación, colaboración, toma de responsabilidades, mantenimiento de una visión compartida, resolución efectiva de problemas, respeto y apoyo a otros, facilitamiento de procesos de interacción y espíritu inquisitivo y experimentador.

Los hallazgos de Porras y Hoffer (1986), sugieren que incrementando la frecuencia de este "estilo de comportamiento laboral", a través de un cambio organizacional planeado, puede generarse un incremento en la efectividad organizacional.

En cuanto a los comportamientos exhibidos por los gerentes, Porras y Hoffer (1986), en la encuesta de los líderes identificaron cinco categorías de comportamiento gerencial, los cuales generan un cambio organizacional exitoso. Estos son: promover el flujo de información, generar participación, liderar a través de la visión, funcionar estratégicamente y desarrollar a los demás.

Dado que los patrones exhibidos por los gerentes pueden ser una influencia importante en las actitudes y comportamientos de sus empleados, ya que éstos tienden a modelar el comportamiento de sus superiores, son particularmente relevantes los hallazgos de Porras y Hoffer (1986) sobre los comportamientos deseables en la gerencia para que un programa específico de cambio organizacional tenga éxito.

Posteriormente, Robertson (1994) efectuó una investigación, en la cual estudió la relación entre el estilo de comportamiento laboral y el comportamiento del empleado, bajo la hipótesis de que determinados comportamientos laborales podían tener importantes implicaciones en el proceso de cambio organizacional planeado.

Tomando como premisa que el desempeño de una organización es una función de los comportamientos agregados de sus miembros, Robertson (1994), plantea que los procesos de cambio organizacional planeado requieren que una masa crítica de la organización esté de acuerdo con un comportamiento diferente. Afirma que es importante identificar estos comportamientos a fin de promoverlos y aumentar las posibilidades de éxito del proceso de cambio planeado.

Los resultados de la investigación mostraron que la efectividad en el logro de las metas esta positivamente relacionado con la frecuencia de cierto estilo de comportamiento laboral; que el comportamiento facilitador de la gerencia está positivamente relacionado con la frecuencia del estilo de comportamiento laboral; la complejidad del trabajo está positivamente relacionada con la frecuencia del estilo de comportamiento laboral; y finalmente que los tres aspectos laborales tienen un efecto interactivo en la frecuencia del estilo de comportamiento laboral.

Este estilo de comportamiento laboral estudiado por Robertson (1994), es 
el planteado por Porras y Hoffer en 1986, es decir, comunicación abierta, colaboración, toma de responsabilidades, mantenimiento de la visión compartida, solución efectiva de problemas, respeto, apoyo, facilitamiento de la interacción de procesos y ambiente inquisitivo y de experimentación. Por su parte el comportamiento facilitador de la gerencia se refiere a promover el flujo de información, generar participación, liderar con visión, trabajar estratégicamente y desarrollar al personal. De estos resultados, se desprende con claridad la importancia de la existencia de valores humanistas para el éxito de los modelos de cambio planeado organizacional, basados en las personas. Y adicionalmente se plantea la importancia de un liderazgo gerencial que apoye el proceso a fin de que éste sea exitoso.

A lo largo de la investigación hemos encontrado reiteradamente ciertos valores humanistas, como presentes en los estudios y aplicaciones en diferentes empresas de distintas partes del mundo. Entre estos valores se encuentran la Delegación de Autoridad (Empowerment), Apertura en la Comunicación, Cultura de Colaboración, Participación y Aprendizaje continuo. A partir del análisis desarrollado, se considera que estos son los valores humanistas más representativos de los modelos de cambio sociotécnicos, requeridos en el entorno actual; $y$ por lo tanto, aquellos que deben alinear el comportamiento para cambiar organizaciones en la Postmodernidad.

\section{Valores requeridos para el cambio organizacional}

Blanchard y O'Connor (1997:73), mencionan que "El éxito verdadero no proviene de proclamar nuestros valores, sino de ponerlos en práctica consecuentemente todos los días". Así que conociendo los valores requeridos, el siguiente paso es saber si en la empresa están presentes o no. Si lo están, debe alinearse la organización alrededor de ellos y si no lo están, entonces habrá que desarrollarlos antes de poner en práctica cualquier otro cambio. Ahora bien, cómo reconocer si se tiene: Delegación, comunicación abierta, participación, cultura de colaboración o aprendizaje continuo? A continuación se analiza cada valor a fin de definir los indicadores de su presencia en la organización.

\subsection{Delegación de autoridad}

Para Stoner et al (1996:389), el delegar facultades no es otra cosa que otorgar a los empleados la libertad y la responsabilidad de efectuar su trabajo de acuerdo con su criterio, sin tener que dirigirse continuamente a autoridades de mayor nivel para conseguir autorización; de manera más formal, señala el autor que, delegación es "El acto de asignar a un subordinado la autoridad formal y la responsabilidad para que realice actividades específicas".

Según Koontz y Weihrich (1998), el proceso de delegación implica: a) Determinación de los resultados esperados de un puesto, b) Asignación de tareas a ese puesto, c) Delegación de autoridad para el cumplimiento de tales tareas y d) Responsabilidad de la persona que ocupa el puesto respecto del cumplimiento de las tareas. En la práctica es imposible separar las diversas partes de este proceso ya que al asignar metas, se debe proveer la 
autoridad para lograrlas y al delegar autoridad hay que dar a conocer los resultados finales en función de los cuales se delega. Agregan estos autores, que aún cuando la elaboración de organigramas, y descripción de metas y deberes administrativos contribuyen a la eficaz delegación de autoridad, ciertas actitudes personales son indicadores de una delegación real:

- Receptividad: Uno de los atributos básicos que debe tener un gerente que delega, es la disposición a conceder que otras personas pongan en práctica sus ideas. La toma de decisiones supone cierto grado de discrecionalidad, de modo que es probable que las decisiones tomadas por un subordinado no sean iguales a las que habría tomado el superior. El jefe debe no solo aceptar de buen grado las ideas de los demás, sino también apoyarlas, colaborar con su puesta en práctica y elogiar su inventiva.

- Disposición a ceder: Para delegar autoridad eficazmente, un administrador debe estar dispuesto a ceder a sus subordinados el derecho a toma de decisiones, aún cuando él podría realizarlas mejor que ellos. En muchas empresas los administradores o dueños de empresas continúan tomando decisiones subalternas cuando ascienden en el nivel jerárquico 0 sus empresas crecen tanto que ameritan la delegación. En este sentido, los administradores deben aplicar la ley de la ventaja administrativa comparativa, es decir que ellos pueden realizar mayores contribuciones a una empresa si se concentran en las tareas más importantes para los obje- tivos de la organización y asignan a sus subordinados sus demás actividades, aún en el convencimiento de que el superior las realizaría mejor que el subordinado.

- Disposición a permitir que los demás cometan errores: La persistente supervisión de los subordinados a fin de evitar que cometan errores, vuelve imposible la verdadera delegación de autoridad. Aún cuando un gerente no va a permitir que alguien de su personal cometa un error que dañe a la compañía o a él mismo, es necesario entender que todas las personas cometen errores y se debe permitir al subordinado cometerlos y considerar su costo como parte de la inversión en desarrollo de personal.

Una paciente asesoría, el planteamiento de preguntas orientadoras y la detallada explicación de los objetivos y políticas, son algunas de las formas que pueden manejarse para una adecuada delegación de autoridad. Las críticas intimidatorias o el insistente señalamiento de sus deficiencias solo consiguen desalentar a los subordinados.

- Disposición a confiar en los subordinados: La delegación de autoridad implica una actitud de confianza entre unos y otros. En ocasiones esta confianza es difícil de conseguir ya que muchos administradores posponen la delegación argumentando que su personal carece de experiencia, es incapaz de manejar subordinados, no ha desarrollado todavía buen juicio o no percibe todos los elementos de una situación. En el caso de que las suposiciones fueran ciertas, el supe- 
rior debe capacitar para delegar; sin embargo en ocasiones se confunde el miedo a ceder y la falta de receptividad con falta de confianza.

- Disposición a establecer y aplicar controles amplios: Para delegar autoridad, se debe estar dispuesto a encontrar los medios para obtener retroalimentación, es decir, asegurarse de que la autoridad está siendo utilizada en apoyo a las metas y planes de la empresa o departamento. Siendo que el jefe nunca puede delegar su responsabilidad, el establecimiento de controles es un imperativo; pero el uso de controles implica la definición de estándares de desempeño, por lo tanto involucra un serio proceso de planificación. La ausencia de una planeación adecuada puede ser, en oportunidades, el motivo de una renuencia a delegar y confiar en el personal.

Cuando en una organización existe delegación de autoridad, los individuos sienten que el trabajo les pertenece, no solo están haciendo lo que se les pide, el trabajo les importa realmente, ellos saben qué tan bien se está haciendo, pueden expresar sus ideas, el trabajo es parte de ellos mismos y ellos tienen control sobre lo que hacen. Todo esto, como planteaba Deming (1989) en sus muy conocidos 14 puntos $^{4}$, hace sentir al traba- jador orgullo por lo que hace y aumenta su eficacia y eficiencia al incrementar su compromiso con la organización.

Ahora bien, ¿podríamos decir que es básico para lograr un cambio organizacional en la gerencia del nuevo milenio, la existencia de delegación de autoridad? Al respecto, los hallazgos de García y DoIan (1997), plantean una nueva realidad gerencial para el siglo XXI, según la cual el paradigma de la modernidad tiene una cultura orientada al control, mientras que el de la postmodernidad tiene una cultura orientada al desarrollo (Ver Cuadro 1).

Si comparamos la visión modernista y postmodernista presentada en el Cuadro 1, con los indicadores de delegación de Koontz y Weihrich (1998), se encuentran las siguientes semejanzas: El control visto a través del autocontrol, autonomía y responsabilidad representa delegar autoridad para que el individuo tome decisiones, sea responsable por ellas y sepa hasta donde puede tomarlas. Es decir, la disposición a ceder mencionada por Koontz y Weihrich (1998).

Aprender de los errores, y estar dispuesto a modificar su manera de pensar, forma parte de la receptividad y de la disposición a que tanto los subordinados como el mismo ejecutivo cometan errores. Confiar en que los demás tienen criterio, forma parte de la disposición a confiar en los subordinados. Y la capacidad

Deming explicaba que una alta productividad no podía venir solamente de una buena "administración de personal", sino que la organización verdaderamente efectiva integraría con éxito las personas al sistema total de operaciones a través de sus 14 puntos: 1. Planificar para el largo plazo; 2. No ser complaciente con la calidad; 3. Establecer controles estadísticos; 4. Proveedores buenos y pocos; 5 . Determinar de origen de los problemas; 6 . Entrenar a los trabajadores para el puesto; 7. Elevar la calidad de los supervisores de línea; 8. Olvidar el temor; 9. Colabora- 


\section{Cuadro 1}

\section{Visión moderna y postmoderna de la delegación de autoridad}

\begin{tabular}{|c|c|c|}
\hline Valores & $\begin{array}{l}\text { Paradigma de la modernidad: } \\
\text { CONTROL }\end{array}$ & $\begin{array}{l}\text { Paradigma de la postmodernidad } \\
\text { DESARROLLO }\end{array}$ \\
\hline Control & $\begin{array}{l}\text { Supervisión orientada a corregir des- } \\
\text { viaciones de la norma }\end{array}$ & $\begin{array}{l}\text { Supervisión orientada a facilitar el auto- } \\
\text { control, autonomía y responsabilidad. }\end{array}$ \\
\hline Apoyo & $\begin{array}{l}\text { Ayudar a disimular errores y alabar fal- } \\
\text { samente ideas de otros. }\end{array}$ & $\begin{array}{l}\text { Ayudar en la ejecución de ideas y } \\
\text { aprender de los errores. }\end{array}$ \\
\hline Integridad & $\begin{array}{l}\text { Intransigencia ante las creencias pro- } \\
\text { pias. Ceder jamás. }\end{array}$ & $\begin{array}{l}\text { Disposición a modificar cuando se cree } \\
\text { estar equivocado o hay posibilidades } \\
\text { de mejorar }\end{array}$ \\
\hline Respeto & $\begin{array}{l}\text { Acatar los razonamientos de otros sin } \\
\text { discutirlos, sobre todo si son jefes. }\end{array}$ & $\begin{array}{l}\text { Confiar en que todos tenemos interés y } \\
\text { capacidad de aprender (aun los jefes) }\end{array}$ \\
\hline $\begin{array}{l}\text { Seguridad en } \\
\text { uno mismo }\end{array}$ & $\begin{array}{l}\text { Mostrar capacidad para ganar, persua- } \\
\text { dir y firmeza, sobre todo con subordina- } \\
\text { dos }\end{array}$ & $\begin{array}{l}\text { Mostrar capacidad para afirmar y so- } \\
\text { meter a juicio los criterios. Ser vulnera- } \\
\text { ble sin sentirse intimidado. }\end{array}$ \\
\hline
\end{tabular}

Fuente: García y Dolan, (1997). Elaboración propia a partir del "Significado de valores esenciales en la antigua cultura orientada al control y en la cultura de futuro orientada al desarrollo".

para someter a juicio los criterios propios y ajenos, estará relacionada con su capacidad de crear controles que retroalimenten la corrección de esos criterios. Es decir, que los indicadores de una delegación real, son a su vez parte de las características de una cultura orientada al desarrollo para el nuevo milenio.

Finalmente puede decirse, que todos los autores mencionados a lo largo de este trabajo, al tratar de explicar las necesidades de la gerencia del nuevo milenio, le dan un factor preponderante a la delegación de autoridad como elemento fundamental para aumentar el compromiso del individuo con la organización y la satisfacción con el puesto, los cuales son dos características básicas de esta generación de trabajadores que comenzó a incorporarse en los 90's, lo que Robbins y Coulter (1996) han llamado: La generación $X$.

\subsection{Apertura en la comunicación}

Peter y Waterman (1984:275:281), comentan que si les pidieran un consejo general para la gerencia, una verdad incontestable que hubieran destilado de su investigación de las empresas excelentes, se sentirían tentados a responder: "Diseñe un sistema de valores" y entre los

ción interdepartamental en vez de competencia; 10 . No a las metas numéricas; 11 . Trabajar con calidad, no solo cumplir horario; 12. Empleados que comprendan lo que hacen; 13. Mantener entrenados a los empleados en las nuevas habilidades según las necesidades que surjan; 14. Responsabilizar a los gerentes por el cumplimiento de los principios. 
valores básicos que ellos encontraron en las empresas excelentes, está el "Creer en la importancia de la informalidad para mejorar las comunicaciones". Dicho de otro modo, que la comunicación sea abierta en todos los sentidos y no solamente la formal y jerárquica plasmada en el organigrama.

Robbins (1996), plantea que existe una relación entre la comunicación y la satisfacción de los empleados: a menor incertidumbre, mayor satisfacción. Las distorsiones, ambigüedades e incongruencias incrementan la incertidumbre $y$, por lo tanto, tienen un impacto negativo sobre la satisfacción.

Mientras menos distorsiones haya en la comunicaciones, la información se recibirá en el sentido y la forma que se pretendió al emitirla. El uso extenso de canales verticales, laterales e informales incrementará el flujo de la comunicación, reducirá la incertidumbre y mejorará el desempeño y satisfacción del grupo. Por otra parte existe una relación positiva entre la comunicación eficaz (la cual incluye factores como la confianza, precisión, deseo de interactuar, receptividad de la administración e información ascendente) y la productividad de los empleados.

Cuando los cambios son más frecuentes y el futuro es más incierto, los empleados desean saber cada vez la lógica subyacente en las decisiones que se están efectuando. En tiempos de estabilidad y empleo permanente, un canal abierto de comunicación no tenía tanta importancia para el personal ya que los cambios no necesariamente los afectarían; pero en estos nuevos tiempos, sin garantía, los empleados quieren saber qué ocurre, por qué y cómo les afectará a ellos. Buscan que la gerencia haga algo por compensar la diferencia entre lo que solían tener garantizado y lo que tienen ahora. Ese algo es mantenerlos informados (Robbins, 1996).

Lee lacocca manifiesta: "La única forma de motivar a las personas es comunicándose con ellas" (Citado por García y Dolan, 1997:157), y esto nunca fue más cierto que en la sociedad postcapitalista, en la sociedad del conocimiento, ya que la principal herramienta para transmitir conocimiento es la comunicación. Las organizaciones ya no son, ni pueden ser verticales; son "orquestas sinfónicas", en la cual, cada ejecutante toca directamente y sin intermediario para el "director", todos hacen cosas diferentes, pero tienen la misma partitura, es decir la misma información, por eso todos trabajan para el mismo objetivo (Drucker, 1994).

Sin una comunicación eficaz en todos los sentidos, no puede darse una de las bases de la organización basada en el conocimiento, como lo es el que todos los miembros deben estar en capacidad de controlar su propio trabajo mediante la retroinformación, de sus resultados, a sus objetivos. Es también responsabilidad de todos los miembros comunicarles sus objetivos, sus prioridades y su proyectada contribución, a sus compañeros de trabajo -hacia arriba, hacia abajo y lateralmente. $Y$ es responsabilidad de todos los miembros asegurarse de que sus propios objetivos concuerden con los de todo el grupo (Drucker, 1994).

Pero para que esto se dé, es necesario "acabar con el miedo" (Deming, 1989), es decir, que el miedo no impida a los empleados hacer preguntas, reportar problemas o manifestar ideas. Una cultu- 
ra de apertura en la comunicación donde los empleados no teman decir la verdad, y los gerentes puedan aprovechar sus talentos, es esencial. Empresas como Federal Express y Motorota han pasado a los anales de la Administración por vivir bajo este valor y basar su éxito en él (Stoner et al, 1996).

La comunicación interna tiene tres direcciones básicas y dos tipos de redes (García y Dolan, 1997; Gibson et al, 1996; Koontz y Weihrich, 1998; Robbins, 1996 y Stoner et al, 1996): Comunicación descendente, ascendente y lateral o cruzada (incluye horizontal y diagonal), y las redes formales e informales (incluye la amistad y los rumores) siendo todas necesarias para la efectividad de la comunicación en la organización.

La comunicación descendente es absolutamente jerárquica, de los niveles superiores a los niveles inferiores. Esta comunicación, en ocasiones es inadecua$\mathrm{da}$, pero necesaria en las organizaciones para girar instrucciones, dar a conocer políticas, normas, reglamentos, entre otros. Este tipo de comunicación, cuando solo usa las redes formales, tiene un proceso muy lento y es necesario contar con un sistema de retroalimentación para verificar si el mensaje ha llegado y si fue percibido tal como lo propuso el emisor.

La comunicación ascendente existe en ámbitos democráticos y participativos; en otro tipo de ambiente es absolutamente ineficaz, ya que es difícil que empleados con miedo, expresen sus ideas 0 informen sobre resultados negativos de su labor. Esta comunicación es indispensable para la toma coherente de decisiones. Además de la cadena de mando, los sistemas de sugerencias, procedimientos de apelación, sesiones de asesoría, reuniones grupales y práctica de puertas abiertas, son ejemplos de este tipo de comunicación.

La comunicación lateral o cruzada, por su parte, incluye el flujo horizontal de información, entre personas de iguales o similares niveles organizacionales y el flujo diagonal, entre personas de diferentes niveles sin relaciones directas de dependencia entre sí. Este tipo de comunicación sirve para acelerar el flujo de la información, procurar una mejor comprensión y coordinar esfuerzos para el cumplimiento de los objetivos organizacionales. Los comités multidisciplinarios, los grupos de trabajo, las relaciones ínter departamentales, entre otros, fomentan este tipo de comunicación cuando se usan las redes formales. En cuanto al uso de redes informales, las amistades y los equipos de juego hacen uso de la comunicación cruzada.

Cuando se habla de apertura en la comunicación, no solo es necesario que la gerencia apoye la existencia de las tres direcciones y los dos tipos de redes, sino que la calidad de esa comunicación incluya indicadores como:

- Confianza: "La confianza es el centro de la comunicación, la colaboración y la disposición a cambiar" (Koontz y Weihrich, 1998:73). Tradicionalmente la confianza se relaciona con la lealtad, cumplimiento de promesas e integridad, pero en la organización el concepto debe ir más allá: hacia una cultura de confianza que trascienda el liderazgo individual. Esa cultura de confianza debe ser mutua entre dirigentes y subordinados de manera que cada uno se comunique con el 
otro en el entendido de que cada quien puede expresar respetuosamente sus ideas sin temer a medidas retaliativas ulteriores (Gibson et al., 1996). La desconfianza, la amenaza y el temor minan la comunicación. En un ambiente en el que estén presentes estos factores, todo mensaje será visto con escepticismo. La desconfianza puede ser producto de incongruencias en la conducta del superior, o de anteriores experiencias en las que el subordinado fue castigado por haberle transmitido honestamente a su jefe información desfavorable pero verídica. Ante esta situación la gente toma una actitud defensiva y se repliega (Koontz y Weihrich, 1998).

- Empatía: Es la capacidad de ponerse en lugar del otro y asumir sus opiniones y emociones, lo que supone ser comunicador y receptor. Con excesiva frecuencia, los dirigentes se consideran mejores comunicadores que lo que opinan sus subordinados. La empatía puede ayudar a un gerente a que se comunique de forma eficaz con sus supervisores y a estos a comprender la posición que el gerente está tratando de transmitir (Gibson et al., 1996). El desarrollar un proceso de empatía lleva a los individuos a escuchar activamente, es decir concentrarse totalmente en el significado que el emisor está tratando de transmitir. Un escucha empático atiende cuidadosamente a lo que se le dice y reserva sus juicios sobre el contenido del mensaje, generando a su vez empatía en el emisor (Robbins y Coulter, 1996).

- Retroalimentación: Cuando un subordinado da una idea o plantea una si- tuación y no obtiene respuesta, se siente defraudado con la organización y su disposición a comprometerse con ella mermará; por el contrario si el individuo recibe respuesta a sus inquietudes, en el futuro estará más dispuesto a involucrarse en los procesos de la organización. Asimismo, si el superior lanza ideas que solo son recogidas por la apatía, su interés en comunicarse con sus subordinados en el futuro se verá opacada. La retroinformación mantiene vivo el deseo de comunicarse entre los miembros de la organización.

En suma, podemos decir que una organización es abierta en la comunicación cuando ésta se da con confianza, empatía y retroalimentación, en todos los sentidos (vertical, horizontal y diagonal) y existen redes tanto formales como informales para expresarse. Esto queda evidenciado en el estudio de Vinten (1999), en el cual todas las empresas exitosas tenían una mezcla de sistemas formales e informales de comunicación y declaraban que no podrían sobrevivir sin ambos, ya que las ventajas de uno no eran compensadas por el otro. Concluye el autor, diciendo que el estudió demostró que las empresas que no tenían un balance entre los dos tipos de comunicación tendían a buscarlo para aumentar su efectividad.

\subsection{Participación}

La participación de los empleados está muy ligada al concepto de Calidad de Vida Laboral, es decir el grado en que los miembros de una organización laboral son capaces de satisfacer importantes necesidades personales a través de sus 
experiencias con la organización. O en otras palabras, un ámbito laboral que estimula el espíritu humano, inspira el crecimiento, desarrolla personal y logra resultados para la organización.

Belcher (1991), plantea una encuesta sobre "Mejor / peor", en la cual los grupos sociales deben identificar las mejores y peores organizaciones donde han estado y por qué las consideran así. Los resultados apuntan a que las mejores organizaciones tenían buen liderazgo, metas comunes, reconocimiento y participación y esto se traducía en trabajo en equipo, colaboración, orgullo de los integrantes, buena comunicación y oportunidad de crecimiento. Por otra parte, la peor organización donde los entrevistados habían estado tenía: mal liderazgo, falta de participación, mala comunicación y baja moral; lo cual se traducía en falta de apreciación, falta de orientación, baja productividad y presión excesiva. Esto indica que la gente se siente cómoda en empresas con calidad de vida laboral, y eso significa, entre otras cosas: Participación.

En los últimos tiempos con el desarrollo de la Calidad Total, se ha teorizado mucho sobre la participación; los círculos de calidad son un ejemplo de técnicas que tienden a estructurar la participación grupal. Pero el verdadero poder de la participación radica en su capacidad para inducir un cambio cultural al fomentar un estilo de gestión participativa y esto no tiene nada que ver con una técnica en particular.

La gestión participativa va más allá; los empleados colaboran en la fijación de metas, planificación de actividades, están informados sobre el rumbo y el desempeño de la organización. Opinan so- bre las decisiones que los afectan y se los educa para que cada vez estén más capacitados para mejorar el desempeño organizacional. El reto de la participación se puede asumir desde tres enfoques diferentes: el de las Relaciones Humanas, el de los Recursos Humanos y el de Alta Participación (Lawler, 1986).

El primero (Relaciones humanas), está basado en la premisa de que la satisfacción laboral incrementa la productividad, por lo tanto, se orienta a elevar la satisfacción laboral y reducir la resistencia al cambio. No reestructura tareas o busca transformar la cultura organizacional.

El segundo enfoque (Recursos Humanos), profundiza más en la participación, a nivel de tarea, integra la participación con el funcionamiento cotidiano de la organización. Respeta al individuo como un recurso valioso dentro de la organización y lo prepara permanentemente para que pueda aportar más e intervenir en la toma de decisiones.

El tercer enfoque (alta participación), se basa en el supuesto de que los empleados sean capaces de tomar importantes decisiones sobre su trabajo y que se obtiene un máximo desempeño organizacional cuando la gente ejerce considerable control sobre sus actividades. Está muy relacionado con el empowerment. Estas organizaciones poseen equipos laborales autosuficientes, son muy horizontales, los sistemas formales están orientados a reforzar la participación y requieren mucho compromiso de los directivos con el cambio. Esta es la verdadera gestión participativa y la más difícil de alcanzar.

Según Belcher (1991), la influencia de los empleados en la toma de decisio- 
nes, en la resolución de problemas, la información compartida, la retroalimentación positiva, y el trabajo estimulante e interesante, son indicadores de la participación de los empleados:

- Toma de decisiones: Cuando los empleados tienen la oportunidad de influir sobre las decisiones que afectan sus tareas, se sienten más dueños de su destino, en vez de engranajes en una maquinaria.

- Resolución de problemas: La activa solicitud de ideas a los empleados para la resolución de problemas aumenta su capacidad para hacer aportes y sentirse orgullosos de sus logros.

- Información compartida: Significa informar continuamente a los empleados acerca de las razones que motivan las decisiones gerenciales, lo cual les da mayor capacidad para reaccionar positivamente ante situaciones cambiantes.

- Retroalimentación positiva: La necesidad de conocer el desempeño personal y los aspectos que deben ser mejorados, es una característica de la naturaleza humana, igualmente es una necesidad la gratificación por un trabajo bien hecho. Adicionalmente, el trabajo interesante y estimulante hace que los trabajadores que son responsables de diversas tareas y disfrutan de cierto grado de autonomía y discrecionalidad obtienen mayor satisfacción laboral que aquellos que realizan tareas repetitivas y tediosas.

Es importante resaltar que, la participación debe ser a todos los niveles, como lo demuestra, Shapiro (2000) realizó un estudio en 14 organizaciones a lo largo de ocho países de la Unión Europea, en el cual quedó en evidencia que las iniciativas de participación y desarrollo de los empleados pueden dificultarse si todos no se involucran de la misma manera. Es frecuente que las organizaciones ofrezcan mayor entrenamiento e información a su personal a tiempo completo que a su personal a medio tiempo, eventual o asesor. Por ende, estos individuos se sienten menos comprometidos con la organización y no se involucran en sus procesos. Adicionalmente otros estudios recientes hacen énfasis en que la participación está íntimamente relacionada con el contexto cultural y la actitud gerencial (Baruch, 1998; Cassar, 1999; Rees y Porter, 1998; Ryan, 1999).

\subsection{Cultura de Colaboración}

Es un lugar común el mencionar a los equipos como pilar fundamental de la nueva gerencia, pero para que existan equipos en la organización se requiere previamente de la existencia de una Cultura de Colaboración. La cultura, se refiere a un sistema de significados compartidos por los miembros de una organización (Robbins, 1996 y Stoner et al, 1996) y uno de los significados que se comparten es la orientación al equipo $o$ al individuo. Las empresas se mueven desde una baja hasta una alta orientación al equipo, es decir desde un bajo hasta un alto espíritu de colaboración entre las partes.

Drucker (1994), plantea que en la sociedad postcapitalista (vale decir: postmoderna) aumentar la productividad de los trabajadores del conocimiento será posible solo escogiendo y estableciendo el tipo apropiado de equipo. Este equipo 
no es el antiguo equipo de béisbol en el cual cada quien tiene su parte y la ejecuta, ni siquiera el equipo de la orquesta sinfónica mencionado, en la cual todos tienen posiciones fijas pero dirigidos por un líder. El nuevo equipo debe funcionar como un partido de dobles en tenis, formado por pocos miembros, en el cual los jugadores ocupan una posición "preferida" en vez de fija, se cubren unos a otros y se acomodan a las habilidades y debilidades de sus compañeros. Es un juego de colaboración, bien calibrado, cuyo rendimiento es mayor que la suma del rendimiento de sus miembros porque aprovecha la fuerza de cada uno de ellos y al mismo tiempo minimiza sus debilidades. Este es básicamente el objetivo de una cultura de colaboración: Potenciar las fortalezas y minimizar las debilidades de los miembros de la organización.

En términos generales, puede decirse que la gerencia tradicional y sobre todo la gerencia occidental, estaba orientada al individualismo, ya que la idea de la colaboración y el trabajo en equipo fue popularizada por los japoneses hacia los años 70's, con los círculos de calidad de Kaoru Ishikawa y el "Kaisen" japonés (Deming, 1989). Hacia 1980 comenzaron a utilizarse en occidente las técnicas japonesas, pero no necesariamente a comprenderse el concepto de colaboración subyacente en el trabajo en equipo.

Una cultura de colaboración implica a) Individuos que no permitan que sus compañeros, superiores o subordinados cometan errores si ellos pueden evitarlo; b) Individuos capaces de compartir la carga de trabajo de otro, sin otra recompensa que ver el trabajo finalizado a tiempo; c) Individuos capaces de compartir sus conoci- mientos, con el objetivo de mejorar el desempeño de otros y de la organización y d) Individuos capaces de tener un liderazgo compartido, dependiendo de las habilidades necesarias en cada caso para la solución de problemas específicos.

Tal vez no haya nada que ejemplifique mejor el espíritu de colaboración, que el tan conocido y comentado ensayo de Pérez Esclarín (1995) sobre el vuelo en formación de "V" que adoptan los gansos cuando vuelan hacia el sur para el invierno:

Los gansos vuelan de esa forma, porque al batir sus alas producen un movimiento en el aire que ayuda al pájaro que viene detrás de él, y volando en $V$ la bandada completa aumenta por lo menos un 71 por ciento más de su poder que si cada pájaro volara solo (sinergia). Yendo en esa formación evitan la resistencia del aire y cada uno se beneficia del trabajo del otro. Cuando un líder se cansa, pasa a uno de los puestos traseros y otro ganso toma su lugar (liderazgo compartido). Los gansos que van detrás graznan para alentar a los que van delante a mantener la velocidad (estímulo).

Finalmente, cuando un ganso se enferma o cae herido, otros dos gansos se salen de la formación y lo siguen para ayudarlo y protegerlo y se quedan con él hasta que está en condiciones de volver a volar o muere; solo entonces vuelven a su bandada o a otro grupo (apoyo). Actuando de esta forma todos los gansos llegan al sur cada año antes de que llegue el invierno. Dicho en otras palabras: trabajando como equipo y colaborando entre ellos logran la meta que se han propuesto de manera oportuna: Son eficaces y eficientes. 


\subsection{Aprendizaje continuo}

Una de las premisas básicas sobre las cuales se sustenta esta investigación, es que la organización debe cambiar para hacerse más competitiva y estar a tono con los nuevos tiempos (globalización, gerencia del nuevo milenio) y uno de los valores que se asevera debe desarrollar la empresa para cambiar es el Aprendizaje Continuo.

Todas las organizaciones aprenden, pero no todas se basan en el aprendizaje, hoy, una gran cantidad se basa en el rendimiento y esto no ha sido malo, hasta ahora, pero en el futuro que está llegando, en la sociedad postcapitalista, en la gerencia del conocimiento, no será suficiente. Fox (1995) plantea que en los últimos treinta años se ha producido más información que en los 5.000 años anteriores y que la cantidad de información disponible en la actualidad se duplica cada cinco años. Por eso Guns y Anundsen (1996), afirman que una organización basada en el rendimiento puede tener una rentabilidad mayor en el corto plazo, pero en el largo plazo, la organización basada en el aprendizaje, tiene mejores posibilidades. La organización basada en el rendimiento se dedica a ejecutar procesos que fueron exitosos en el pasado, pero la organización que aprende, basa sus procesos en lo que se necesitará en el futuro.

Guns y Anundsen (1996:16) señala que una organización de rápido aprendizaje averigua con mayor rapidez lo que funciona mejor de sus competidores, de esta manera obtiene y conserva la ventaja competitiva o sea, la capacidad de generar y conservar sus utilidades y su lugar en el mercado. Más formalmente define aprendizaje organizacional como "Adqui- rir y aplicar los conocimientos, técnicas, valores, creencias y actitudes que incrementan la conservación, el crecimiento y el progreso de la organización".

Por su parte García y Dolan (1997:151), opinan: "una empresa que aprende es aquella capaz de desaprender valores, supuestos y pautas de conducta del pasado para adoptar otros nuevos más eficaces para conseguir sus objetivos de futuro". Agregan, que representa una empresa cuya cultura y liderazgo promueven el aprendizaje continuo de todos sus miembros a todos los niveles, legitimando el diálogo interpersonal de calidad en todas direcciones y transformándose continuamente para adaptarse a las demandas cambiantes del entorno e integrar sus nuevas tensiones internas.

Estos autores, aseguran que existen siete características que permiten reconocer a una empresa que aprende: 1) La capacidad de desaprender, es decir la capacidad de reemplazar viejos hábitos por otros más efectivos en el nuevo entorno; 2) La facilidad de diálogo interpersonal; 3) La visión global de la empresa, vale decir ese flujo de información que permita a los integrantes ver tanto los árboles como el bosque; 4) La eliminación de territorios cerrados entre departamentos (física y conceptualmente); 5) Los equipos autónomos y confiables con legitimidad ante la organización; 6) El apoyo gerencial demostrando entusiasmo ante los cambios y 7) La legitimación del aprendizaje a través de los errores; reemplazando el castigo por la visión de los errores como fuente de innovación (García y Dolan, 1997).

Algunos de estos indicadores del aprendizaje, están presentes en otros valores ya que hay una gran interrelación entre 
ellos. Sin embargo, la capacidad de desaprender, la visión global y el carácter legítimo del aprendizaje a partir de los errores, el cual permite y estimula la innovación y la búsqueda de mejores formas de hacer las cosas, podrían plantearse como los indicadores primordiales de este valor.

A manera de síntesis, y una vez analizado cada uno de los cinco valores básicos, definidos como necesarios para efectuar cambios organizacionales en la postmodernidad, pudimos especificar 20 indicadores de la presencia de ellos. Es- tos indicadores son particularmente importantes en la praxis organizacional, ya que a través de ellos, se puede determinar la existencia o no de los valores indicados en la cultura de la organización. Además, una vez efectuado el diagnóstico (en caso de no estar presentes los valores), los indicadores permitirán conocer cuáles son los aspectos que hay que modificar y/o profundizar para alinear a la organización a través de los valores humanistas determinados. En el Cuadro 2 se presenta un resumen de los valores y sus indicadores.

\section{Cuadro 2}

Valores necesarios en la postmodernidad y sus indicadores

\begin{tabular}{cl}
\hline Valores & Indicadores \\
\hline DELEGACIÓN & Receptividad \\
DE & Disposición a ceder \\
AUTORIDAD & Disposición a permitir que los demás cometan errores \\
& Disposición a confiar en los subordinados \\
& Disposición a establecer y aplicar controles amplios \\
& \\
APERTURA & Comunicación vertical \\
EN LA & Comunicación cruzada \\
COMUNICACIÓN & Confianza \\
& Empatía \\
& Retroalimentación \\
PARTICIPACIÓN & Toma de decisiones \\
& Resolución de problemas \\
& Información compartida \\
& Retroalimentación positiva \\
CULTURA & Compartir labores \\
DE & Compartir conocimientos \\
COLABORACIÓN & Apoyo mutuo \\
& \\
\hline FRENDIZAJE & Capacidad de desaprender \\
& Legitimidad del aprendizaje a través de los errores \\
\hline
\end{tabular}

Fuente: Elaboración propia. 


\section{Conclusiones}

La idea de que el comportamiento organizacional puede ser moldeado deliberadamente a través de un grupo de características laborales, tiene implicaciones muy importantes para los interesados en la planeación de cambios organizacionales.

En el pasado, y sobre todo en nuestros países latinoamericanos donde solemos importar modelos diseñados en otras culturas, los intentos de cambio organizacional tienen un alto índice de fracasos y con mucha frecuencia, no pasan de ser un mero maquillaje: superficial y efímero.

A lo largo del siglo pasado, las organizaciones sufrieron los embates de modelos de cambio estructurales al principio, tecnológicos después, y muchos, pero muchos fracasos debidos a la creencia de que los individuos de acoplarían a estos cambios, ignorando la importancia del componente humano y la resistencia al cambio. De hecho, hasta los años 70 no se había acuñado el término de cultura organizacional, a lo sumo se hablaba de clima organizacional.

Pero a medida que avanzan los tiempos nos encontramos en un mundo interconectado, interdependiente y transdisciplinario, los compartimientos estancos ya no existen, han fracasado los modelos reduccionistas en todas las áreas, incluso en el intento de desarrollar nuestros países. La Postmodernidad se abre paso y se expresa en la necesidad de un desarrollo sustentable, de una literatura sin cortapisas, de un arte no encasillado en escuelas, y sobre todo, de una gerencia no restringida a estructuras y avances tecnológicos.
Ser competitivo implica ahora, no solo tener mayor y mejor tecnología, o cambiar constantemente la estructura; eso no es suficiente, implica organizaciones flexibles con individuos que aprendan más rápido y de manera permanente, que trabajen integrados, jefes que sin dejar su responsabilidad, tengan suficiente confianza en sus subordinados como para delegarles toma de decisiones, y empleados con suficiente confianza en la organización como para participar, innovar y cambiar. Implica un flujo de información en todos los sentidos que permita estar delante de la competencia, quedan atrás los secretos y el guardarse información como herramienta de poder. El conocimiento, y por ende el ser humano que lo posee, es el recurso más importante en las organizaciones.

Ante esta realidad, los cambios organizacionales tienen que girar alrededor del individuo y de los valores que pueden moverlo hacia la nueva cultura (delegación de autoridad, comunicación abierta, participación, colaboración y aprendizaje continuo).

El diagnóstico organizacional a través de estos valores permitirá conocer si están sentadas las bases para comenzar el camino del cambio organizacional, o si por el contrario, tiene que, como paso previo, iniciarse un proceso de alineación integral de esos valores a fin de modificar la cultura y hacerla proclive al cambio, disminuyendo la resistencia e incrementando las posibilidades de éxito, con una reducción de costos, tiempo y esfuerzo en la consecución de los objetivos finales que se plantearon. 


\section{Referencias Bibliográficas}

Baruch, Yehuda, (1998), Applying empowerment: organizational model, Career Development International, Vol. 3, No. 2, pp. 82-87. United Kingdom.

Blanchard Ken y O'Connor Michael (1997), Administración por Valores, Editorial Norma, Colombia.

Belcher, John (1991), Productividad Total, Editorial Granica, Argentina.

Bennis, Warren (1973), Desarrollo Organizacional: su naturaleza, sus orígenes y perspectivas, Fondo Educativo Interamericano, México.

Brown, William y Dodd, Nancy (1998), Utilizing organizational culture gap analysis to determine human resource development needs, Leadership \& Organizational Development Journal, Vol. 19, No. 7, pp. 374-385. United Kingdom.

Buela, Alberto (1997), La Postmodernidad, Razón Española № 82, marzo-abril 1997, Madrid.

Burke, Ronald (1997), Cultural values, satisfaction and performance in a professional services firm Training for Quality, Vol. 5, No. 3, pp. 100-105. United Kingdom.

Cassar, Vincent (1999), Can leader direction and employee participation co-exist? Investigating interaction effects between participation and favorable work-related attitudes among Maltese middle-managers, Journal of Managerial Psychology, Vol. 14, No. 1, pp. 57-68. United Kingdom.

Chiavenato, Idalberto (1995), Introducción a la Teoría General de la Administración, 4a․ Edición, Mc. Graw Hill, Colombia.

Daft, Richard (1998), Organizational Theory and Design, 6a. Edición, Southwestern, Cincinnati, Ohio. USA
Deming, Edward (1989), Calidad, Productividad y Competitividad: la salida de la crisis, Díaz de Santos, Madrid.

Drucker, Peter (1994), La sociedad PostCapitalista, Grupo Editorial Norma, Colombia.

Feurer, Rainer y Chaharbaghi, Kazem (1995), Achieving a balanced value system for continuity and change, Management Decision, Vol. 33, No. 9, pp. 44-51. United Kingdom.

Fox, Janet (1995), Conquer Information Anxiety, Amtrak Express, Vol marzo-abril, p. 10. North Carolina, USA.

French Wendell y Bell Cecil (1996), Desarrollo Organizacional, (5⿳a edición) Prentice Hall, México.

Friedlander, Frank (1976), OD reaches adolescence: an exploration of its underlying values, Journal of Applied Behavioral Science, Vol 12, No. 1, pp 7-12. Texas, USA.

García Salvador y Dolan Shimon (1997), La Dirección por Valores. McGraw Hill, España.

Gellerman, William, Frankel, Mark, y Ladenson Robert (1990), Values and Ethics in Organizational and Human Systems Development: Responding to Dilemmas in Professional Life, Jossey-Bass, San Francisco. USA.

Gibson James, Ivancevich John y Donnelly James (1996), Las Organizaciones, Comportamiento, Estructura, Procesos (8ª . Edición) Mc Graw Hill , Colombia.

Grieves, Jim (2000), Navigating change into the new millennium: themes and issues for the learning organization, The Learning Organization, Vol. 7, No. 2, pp. 54-74. United Kingdom.

Guns, Bob y Anundsen, Kristin (1996), Aprendizaje Organizacional, Prentice Hall, México. 
Harung, Harald Y Dahl Tor (1995), Increased productivity and quality through management by values: a case study of Manpower Scandinavia, The TQM Magazine, Vol. 7, No. 2, pp. 13-22. United Kingdom.

Hurley, Robert, Church, Allan, Burke Warner, y Van Eynde, Donald (1992), Tension, change and values in OD, OD practitioners, Vol. 29, pp. 1-5. New Jersey, USA.

Kahn, Robert (1976), Organizational development: some problems and proposals, Journal of Applied Behavioral Science, Vol 10, No. 2, pp. 485-502. Texas, USA.

Koontz, Harold y Weihrich, Heinz (1998), Administración, una perspectiva global, (11a. Edición), Mc Graw Hill, México.

Lawler, Edward (1986), High Involvement Management, Jossey-Bass Publishers, San Francisco. USA.

Lloyd, Bruce y Kidder, Rushworth (1997), Ethics for the new millennium Leadership \& Organizational Development, Vol. 18, No. 3, pp. 145-148. United Kingdom.

London, Manuel (1999), Principled leadership and business diplomacy. A practica, values-based direction for management development, Journal of Management Development, Vol. 18, No. 2, pp. 170-192. United Kingdom.

Margulies, Newton y Raia, Anthony (1978), Conceptual Foundation of Organizational Development, McGrawHill, New York, USA.

Martínez Miguelez, Miguel (2003), Perspectiva epistemológica de la bioética, Estudio solicitado por la UNESCO para la "I Reunión de la Red Latinoamericana de Bioética", y presentado en Cancún (México), 1-2 Mayo.
Pérez Esclarín, Antonio (1995), Educar, Valores y el Valor de Educar. Parábolas, Editorial San Pablo, Venezuela.

Peter Thomas y Waterman Robert (1984), En busca de la excelencia, editorial Norma, Colombia.

Porras, Jerry y Hoffer, Susan (1986), Common Behavior Changes in Successful Organization Development Efforts, Journal of Applied Behavioral Science, Vol. 22 No. 4, pp. 477-494. Texas, USA.

Rees, David y Porter, Christine (1998), Employee participation and managerial style (the key variable), Industrial \& Comertcial Training, Vol. 30, No. 5, pp. 165-170. United Kingdom.

Robertson, Peter (1994), The relationship between Work Setting and Employee Behaviour: A Study of a Critical Linkage in the Organizational Change Process, Journal of Organizational Change Management, Vol 7, No. 3, pp. 22-43. United Kingdom.

Robbins, Stephen (1996), Comportamiento Organizacional (teoría y práctica) 7ª . Edición, Prentice Hall, México.

Robbins, Stephen y Coulter, Mary (1996), Administración, $5^{\circ}$ edición, Editorial Prentice Hall, México.

Ryan, Margaret (1999), The role of social process in participative decision making in an international context, Participation \& Empowerment: An International Journal, Vol. 7, No. 2, pp. 3342. New York.

Shapiro, Gillian (2000), Employee involvement: opening the diversity Pandora's Box?, Personnel Review, Vol. 29, No. 3, pp. 304-323. United Kingdom

Stoner James, Freeman Edward, Gilbert Daniel (1996), Administración, 6aㅗ edición, Prentice Hall, México. 
Vinten, Gerald (1999), Corporate communications in smal- and medium- sized enterprises, Industrial and Commercial Training, Vol 31, No. 3, pp. 112119. United Kingdom.

Warwick, Dianne y Thompson, Jane (1980), Still crazy after all these years Trai- ning and Development Journal, Vol 34, pp. 16-32. United Kingdom.

Wooten, Kevin y White, Louis (1999), Linking OD's philosophy with justice theory: postmodern implications, Journal of Organizational Change management, Vol. 2, pp 7-20. United Kingdom. 